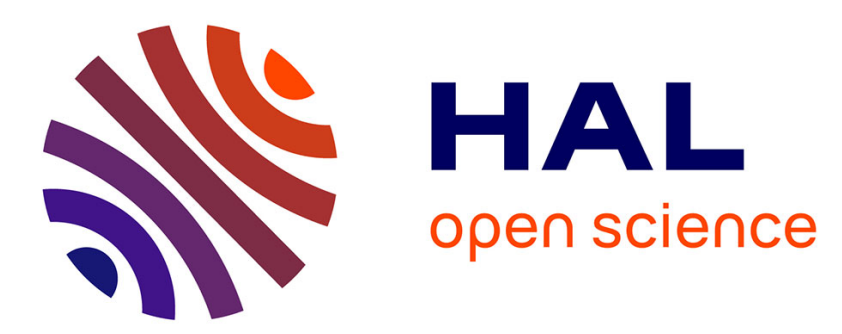

\title{
Contaminant-free end-capped and single-mode acetylene photonic microcell for sub-Doppler spectroscopy
}

Thomas Billotte, Matthieu Chafer, Martin Maurel, Foued Amrani, Frédéric

Gérôme, Benoit Debord, Fetah Benabid

\section{- To cite this version:}

Thomas Billotte, Matthieu Chafer, Martin Maurel, Foued Amrani, Frédéric Gérôme, et al.. Contaminant-free end-capped and single-mode acetylene photonic microcell for sub-Doppler spectroscopy. Optics Letters, 2021, 46 (3), pp.456-459. 10.1364/OL.412507 . hal-03222143

HAL Id: hal-03222143

https://hal.science/hal-03222143

Submitted on 10 May 2021

HAL is a multi-disciplinary open access archive for the deposit and dissemination of scientific research documents, whether they are published or not. The documents may come from teaching and research institutions in France or abroad, or from public or private research centers.
L'archive ouverte pluridisciplinaire $\mathbf{H A L}$, est destinée au dépôt et à la diffusion de documents scientifiques de niveau recherche, publiés ou non, émanant des établissements d'enseignement et de recherche français ou étrangers, des laboratoires publics ou privés. 


\title{
Contaminant-free end-capped and single-mode acetylene photonic microcell for sub-Doppler spectroscopy
}

\author{
Thomas Billotte, ${ }^{1}$ Matthieu Chafer, ${ }^{1,2}$ Martin Maurel, ${ }^{1,2}$ Foued Amrani, \\ 1,2 Frederic Gerome, ${ }^{1,2}$ Benoit Debord, ${ }^{1,2}$ Fetah Benabid ${ }^{1,2}$ \\ ${ }^{1}$ GPPMM Group, Xlim Research Institute, CNRS UMR 7252, University of Limoges, 87000 Limoges, France \\ ${ }^{2}$ GLOphotonics SAS, 123 avenue Albert Thomas 87060 Limoges Cedex \\ *Corresponding author:f.benabid@xlim.fr
}

Received XX Month XXXX; revised XX Month, XXXX; accepted XX Month XXXX; posted XX Month XXXX (Doc. ID XXXXX); published XX Month XXXX

\begin{abstract}
We report on the development of acetylene filled photonic microcell based on an assembly process that is contaminant free and requires no helium buffer-gas nor gluing procedure. The microcell consists of a 7 meter-long and $30 \mu \mathrm{m}$ core-diameter inhibited-coupling guiding hollow-core photonic crystal fiber filled with acetylene gas at a pressure in the range of $80 \mu \mathrm{bar}$, sealed by capping its ends with fusion-collapsing a glass-tube sleeve, and mounted on FC connectors for integration. The microcell shows a robust single-mode behavior and a total insertion loss of $\sim 1.5 \mathrm{~dB}$. The spectroscopic merit of the formed microcell is tested by generating electromagnetic induced transparency and saturated absorption on R13 and P9 absorption line respectively. The sub-Doppler transparencies show a close to transit-time limited linewidth of $17 \pm 3 \mathrm{MHz}$. The latter was monitored for over 3 months. As a demonstration, the microcell was used to frequency-stabilize a laser with fractional frequency instability improvement by a factor 50 at $100 \mathrm{~s}$ integration-time compared to free running laser operation.
\end{abstract}

http://dx.doi.org/10.1364/OL.99.099999

An all-fiber photonic microcell (PMC) is a length of hollowcore photonic crystal fiber (HCPCF) filled with a given gas and whose ends are sealed in hermetic fashion to a solid optical fiber [1]. This new type of gas cell was used to generate sub-Doppler spectral line via electromagnetically induced transparency (EIT) or saturated absorption (SA) in acetylene gas despite the weak strength of its optical transitions [1-8], and to act as an all-fiber optical frequency reference for telecom [1-3] or as a device for slow light applications [9].

Several assembly and sealing techniques were reported [35,10]. The initial fabrication method, which the most commonly used, relies on splicing all-solid optical fibers (e.g. SMF or LMA fiber) to the tips of a gas-filled HCPCF. Such a process allows the PMC to be FC/APC connected for an easy integration. Achieving such low gas pressure cells is made possible by the use of above atmospheric-pressure helium (He) during the splicing process to avoid air pollution in the fiber core. The buffer gas is then evacuated from the fiber core by diffusion through the silica walls of the fiber cladding [4]. However, this technique is not immune from residual gas contamination due to the exposure to the ambient air during splicing. Additionally, the splicing based all-fiber PMC technique is not adapted to all types of fibers. It requires prior fiber-post-processing to match the mode-fielddiameters (MFD) of the two fibers. Indeed, whilst the technique achieves good PMCs with photonic bandgap (PBG) guiding HCPCF (PBG-HCPCF) [11] spliced to SMF, it proved more challenging with inhibited-coupling (IC) guiding HCPCF (IC-HCPCF) [11] because of their larger fiber core and fiber outer-diameter. A reported example of such a postprocessing was Kagome IC-HCPCF tapering to adapt its MFD to that of solid-core fiber [8]. This additional step complicates the PMC assembly process, and induces higher and asymmetric splice-loss. Recently, Curtis et al. [6] devised the insertion of LMA fiber between an IC-HCPCF and an SMF to form a $\mathrm{CO}_{2}$ PMC operating at $2 \mu \mathrm{m}$. However, the insertion loss was found to be $8.5 \mathrm{~dB}$. An alternative to the above splicing technique is based on encapsulating the HCPCF ends in a small chamber made from borosilicate capillary [3]. Here, He gas is not required but this PMC assembly process uses epoxy glue, which is a source of contamination through outgassing, and limit its operating temperature. This limit is exemplified by the observed line broadening from $37 \mathrm{MHz}$ to $77 \mathrm{MHz}$ after 230 days from the PMC assembly [3]. Furthermore, capillary chamber remains relatively bulky and requires free-space optics for light in- and out-coupling. Here, we report on an acetylene PMC assembly technique that is contaminant-free and without the need for the use of 
He gas or gluing. The resulted PMC, equipped with standard fiber connectors for integration, shows a total and symmetric insertion loss of $1.5 \mathrm{~dB}$, and robust single-mode behavior. The PMC airtightness was tested by inspecting SA linewidth and contrast over more than 3 months. Its potential for coherent optics and frequency standards applications was demonstrated by generating EIT and SA, along with laser frequency stabilization.

Figure 1(a) schematically summarizes part of the PMC assembly process. A $30 \mu \mathrm{m}$ core-diameter and $220 \mu \mathrm{m}$ outerdiameter IC-HCPCF, and exhibiting a transmission loss of 10 $\mathrm{dB} / \mathrm{km}$ in 1500-1550 $\mathrm{nm}$ wavelength range, was, at first, baked and pumped to remove any residual gas from the fiber fabrication process. Both ends of a $7 \mathrm{~m}$ long section of the ICHCPCF were then tightly sleeved with a $650 \mu \mathrm{m}$ outerdiameter borosilicate capillary in a manner where the capillary extends by a few mm from the IC-HCPCF end. This was achieved by fusion splicing the capillary with IC-HCPCF at a short section $(\sim 1 \mathrm{~mm})$ located at the fiber end (see the diagram in Fig. 1(b)). The choice of the borosilicate was motivated by its lower softening temperature compared to the fiber silica, and thus to keep the IC-HCPCF physical integrity during the splicing process. The long fiber length is motivated to ensure sufficiently high contrast of the Doppler broaden line with relatively low pressure (10 $\mu \mathrm{bar}$ level). After the above, one sleeved fiber-end is collapsed by fusion to form an end-cap. The latter is cleaved and then etched to a thickness of $\sim 100 \mu \mathrm{m}$ and polished (Fig. 1(b)) after having been mounted on FC/APC connector. The light coupling through this end-cap was found to be $75 \%$, and the light output intensity profile from it shows a Gaussian-like shape. The second sleeved fiber-end is mounted in a manner schematically presented by Fig. 1(a). A length of the same ICHCPCF is attached to a mechanical cell for gas loading and vacuum pumping from one end. The second end is inserted into the capillary of the non-capped end of the $7 \mathrm{~m}$ long ICHCPCF, and spliced to the sleeve in similar fashion as above. The gap between the two IC-HCPCF ends is adjusted so to ensure sufficient light coupling between the two fibers and to later fusion-collapse the borosilicate without damaging the IC-HCPCF end. The fiber is then gone through a sequence of loading and pumping acetylene until the absorption line (here P9) has reached a Doppler linewidth and contrast that matches with the target pressure [4,12]. Once this is achieved, we proceed with the second end-capping following the same above-mentioned fusion-collapse procedure.

Figure 2(a) shows the PMC typical transmission spectrum centered on P9 line $\mathrm{C}_{2} \mathrm{H}_{2}$ absorption at the vicinity of 1530 nm before (blue curve) and after (orange curve) the second end-capping. The oscillations in the blue curve are due to etalon effects from one of the free-space optics, which is corroborated by their disappearance in the PMC absorption trace. The dotted black curve corresponds to Hitran [12] simulated absorption line, giving a gas pressure of $80 \mu \mathrm{bar}$, in good agreement with the recorded pressure during the PMC assembly. Figure 2(b) shows a photography of the formed PMC once mounted on FC/APC connectors. We found the total insertion loss to be $\sim 1.5 \mathrm{~dB}$. Figure 2 (c) summarizes the PMC output beam profile and its stability upon motion. The top of Fig. 2(c) shows the reconstructed near-field intensity, which shows a Gaussian-like shape. In order to test single mode operation of the $\mathrm{PMC}$, we recorded the above intensity profile while hand-shaking the PMC (see visualization 1 in supplementary material).

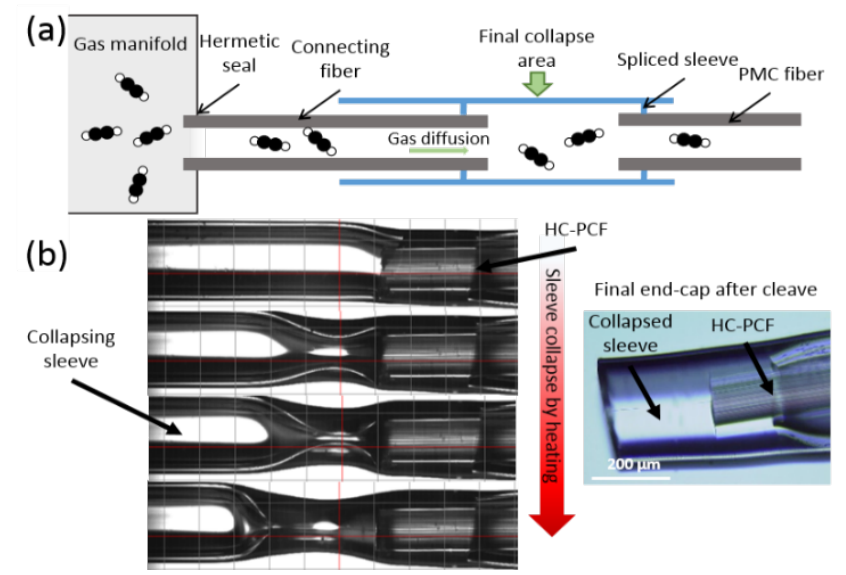

Fig. 1. (a) Set-up scheme of the gas injection into the HCPCF and encapsulation technique. (b) Microscope picture of the end-cap: sleeve during heating collapsing on the HCPCF (on the left), collapsed and cleaved glass sleeve at the end of the HCPCF (on the right).

The extracted peak centroid position and full width at half maximum (FWHM) of the intensity profile show no measurable change to the peak center within the $0.5 \mu \mathrm{m}$ pixel-size of our camera, and a FWHM of $19.6 \mu \mathrm{m}$ compared to $22 \mu \mathrm{m}$ for static position. Such a variation in FWHM correspond to the contribution of higher order modes (HOM) that are excited during the motion. According to [13], the measured relative FWHM difference suggests an HOM contribution to the modal content of less than $-20 \mathrm{~dB}$. The robustness of the PMC guided mode is very promising for onboard and mobile applications. Such a modal property is explained by the IC-HCPCF new cladding design reported in [13].
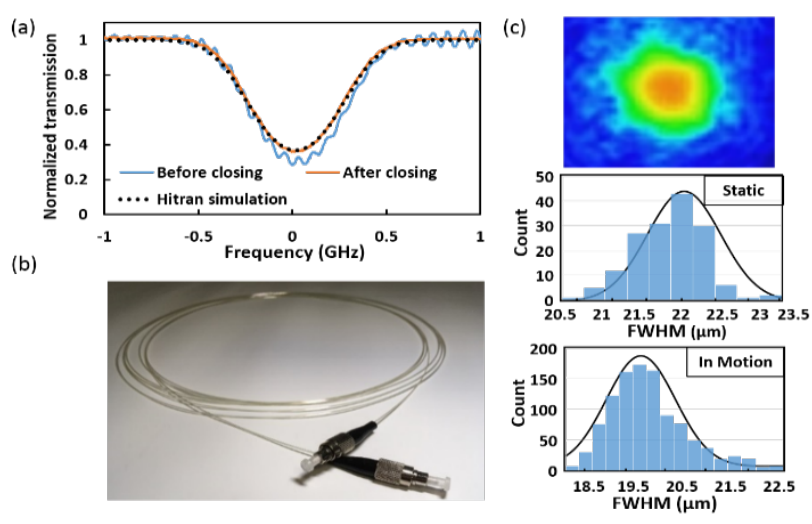

Fig. 2. (a) Doppler limited spectrum before sealing (blue curve), after sealing (orange curve). Comparison with simulated curve (from Hitran database) for $80 \mu$ bar of acetylene (doted curve) and 
7 m-long fiber. (b) Final PMC with polished FC/APC connectors. (c) Near field imaging of polished connectors of the PMC, FWHM of the Gaussian mode static (middle), with fiber motion (down).

Figures 3 and 4 show the developed PMC performance in sub-Doppler spectroscopy by generating EIT (Fig. 3) and SA (Fig. 4). The EIT was generated using a pump laser emitting at $1532.83 \mathrm{~nm}$ and resonant with $\mathrm{P} 13 \mathrm{C}_{2} \mathrm{H}_{2} \quad v_{1}+v_{3}$ rovibrational absorption line, and a probe laser with a frequency-sweeping centered at $1518.21 \mathrm{~nm}$ (R13 line). The pump and probe beam were injected in the PMC in a copropagation configuration. Figure 3(a) (blue curve) shows the typical measured PMC transmission spectrum of the probe for $100 \mathrm{~mW}$ of pump power. The dashed red curve is a fit of the measured Doppler-limited absorption profile. The trace clearly shows the EIT peak centered in the middle of the R13 absorption line, while Fig. 3(b) shows a close-up spectral trace. The EIT origin of this sub-Doppler signal is corroborated by observing its frequency shift when the pump frequency is shifted. The EIT contrast is defined by the ratio of the EIT peak height, $H$, (see Fig. 3(a)) over the Doppler R13 absorption depth, $A$. The EIT linewidth $\Delta v_{E I T}$, is taken to be the FWHM as shown in Fig.3 (b). $H$ and $\Delta v_{E I T}$ were extracted by fitting them with the expressions reported in [14]. The evolution of the contrast and FWHM with pump power coupled into the $\mathrm{HCPCF}, P_{\mathrm{c}}$, has been recorded over a range of 40-205 $\mathrm{mW}$. Here, the fiber loss is neglected and an in-fiber coupling efficiency of 75\% was assumed. Figure 3(c) shows the measured contrast (solid orange diamond symbols) and FWHM (solid blue triangle symbols) versus $P_{c}$. Within the coupling power range, the contrast increases with the coupling power from $10 \%$ to $31 \%$. The FWHM ranges from $17 \mathrm{MHz}$ to $30 \mathrm{MHz}$. The measured data fit well with the theoretical expressions (solid lines) $[14,15]$. The minimum linewidth (FWHM) achievable is $\Delta v_{t t}=(0.58 / \pi)\left(2 v / d_{m}\right)$ [16], set by the transit-time limit and where $v$ being the molecular most probable velocity and $d_{m}$ the guided MFD. The latter is related to the fiber geometrical diameter $d_{f}$ as $d_{m} \approx 0.73 d_{f}$ [17]. For the present acetylene-filled fiber, this linewidth limit due to the transit time is $\sim 14.7 \mathrm{MHz}$ (dashed blue horizontal line). The theoretical EIT linewidth expression can be written as $\Delta v_{E I T}=2 \Gamma_{g r}+\alpha P_{c}$, where $\alpha$ is quantity related to the optical power broadening and the coupling transition dipole moment. $\Gamma_{g r}$ is the sum of all the decoherence terms of the two ground states of the EIT transitions. These are chiefly set by the pressure dependent collisions molecule-molecule, $\Gamma_{\text {coll }}$, molecule-wall, $\Gamma_{\text {wall }}$, and by the laser linewidth broadening contribution, $\Gamma_{\text {laser }}$, i.e. $\Gamma_{g r} \approx \Gamma_{\text {wall }}+\Gamma_{\text {coll }}+\Gamma_{\text {laser. }}$. For a gas pressure of $80 \mu$ bar and using the expressions reported in [14], we have the following HWHM contribution $\Gamma_{\text {wall }}=6.6 \mathrm{MHz}, \Gamma_{\text {coll }}=0.5 \mathrm{MHz}$ and $\Gamma_{\text {laser }} \approx\left(\sqrt{\Delta v_{c}{ }^{2}+\Delta v_{p}{ }^{2}}\right) / 2 \approx\left(\Delta v_{p}\right) / 2=2 M H z$. Thus giving a contribution to the linewidth broadening of $\sim 18 \mathrm{MHz}$. The linear fit shown in Fig. 3(c) shows a linewidth intercept of $18 \pm 3 \mathrm{MHz}$, which is in good agreement with $\Gamma_{g r}$ deduced above. Within the uncertainty of our measurements, this result is an indicator that the in-fiber gas pressure and content hasn't been altered during the PMC assembly.
The suitability of the PMC for coherent optics and frequency reference applications was further investigated by performing SAS (saturable absorption spectroscopy) on P9 absorption line, 29 days after closing the PMC. The experimental set-up to generate the SA sub-Doppler line is similar to the one described in [2]. A tunable laser is set so its frequency probes the P9 line, and is amplified with an EDFA. The laser beam is split into a pump and probe beams using $90 / 10$ divider. The frequency of the probe is down converted by $55 \mathrm{MHz}$ shift to avoid interference of the probe signal with the pump backscattered signal.
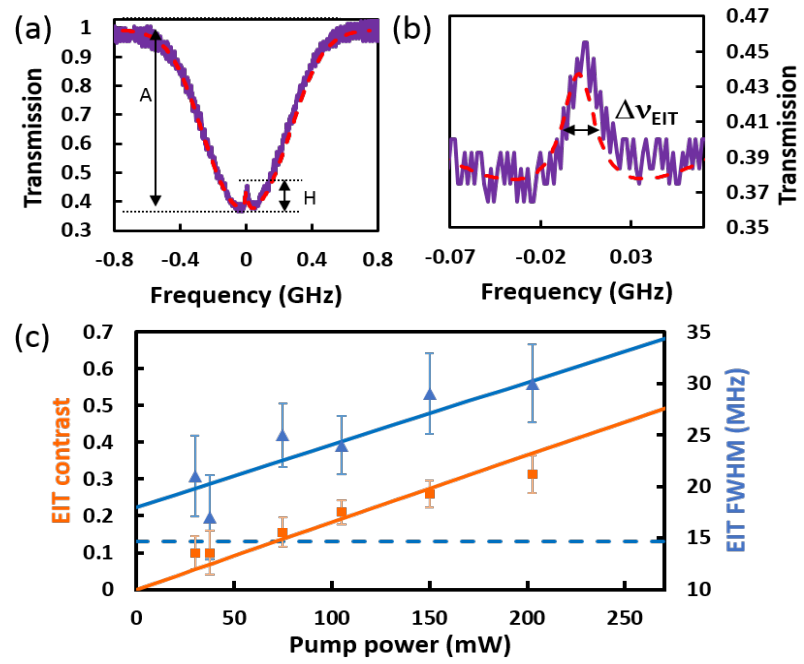

Fig. 3. (a) EIT measurement for pump power of $100 \mathrm{~mW}$ with fit in dashed red line. $A$ is the absorption depth and $H$ is the EIT peak height. (b) Zoom on the EIT peak (c) Experimental EIT contrast $\left(\mathrm{H}_{E I T}\right)$ and EIT FWHM $\left(\Delta v_{E I T}\right)$ versus Rabi frequency (dots) and simulated curves (line). The dashed line correspond to the asymptote of the EIT FWHM.

Figure 4 (a) shows the typical measured PMC transmission spectrum of the probe for $145 \mathrm{~mW}$ of coupled pump power, $P_{\mathrm{p}}$, (blue curve) and its theoretical fit (dashed red curve) [16]. Figure 4(b) shows the spectra close-up around the SAS subDoppler line. Figure 4(c) shows the measured SA line contrast and the FWHM (blue symbols) and their respective theoretical fits with $P_{\mathrm{p}}$ when it is increased from $23 \mathrm{~mW}$ to $145 \mathrm{~mW}$. The contrast increases from $6 \%$ to $25 \%$, and the FWHM ranged from 17 to $20 \mathrm{MHz}$.

Similar to EIT, the SA linewidth broadening is dominated by collisions, hence, the SA sub-Doppler linewidth evolution with the pump power follows the expression $\Delta v_{S A} \approx$ $2 \Gamma_{g r} \sqrt{1+P_{p} / P_{s}}$, where $P_{s}$ is the saturation power [16]. The theoretical evolution of the linewidth is in a good agreement with the experimental data leading us to an intercept value of $17.5 \pm 3 \mathrm{MHz}$ similar to the value found with EIT campaign. It is noteworthy, that with P14 line a contrast as high as 30\% and a FWHM of $19 \mathrm{MHz}$ was obtained with a pump power of $145 \mathrm{~mW}$.

To ensure the PMC remains tightly sealed and that no contaminant is introduced, the SA FWHM measurements have been recorded until the $100^{\text {th }}$ day since the PMC full assembly. Figure 5(a) shows the measured P9 line SA FWHM 
from day 29 to day 100 . Here, the pump power was set at 23 $\mathrm{mW}$. The data shows an average FWHM of $17.2 \mathrm{MHz}$ and standard deviation of $1 \mathrm{MHz}$, which is well within our measurement uncertainty, thus indicating a stable FWHM, and in turn, a constant gas pressure of the PMC.

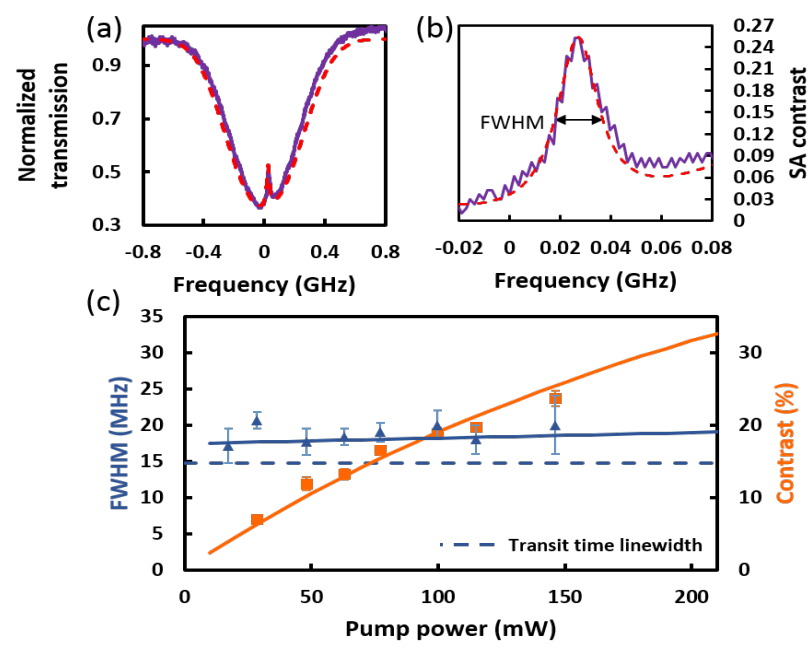

Fig. 4. (a) Normalized SAS measurement on P9 absorption line of acetylene (full purple line), numerical calculation for a pressure of 80 $\mu$ bar (dashed red line) for a pump power of $145 \mathrm{~mW}$. (b) SA contrast zoomed on SA peak with the numerical calculation in dashed line. (c) Experimental (dots) and theoretical (line) SA transparency peak contrast and linewidth evolution with pump power. The dashed line corresponds to the transit time broadening.

As a proof of concept, the developed acetylene PMC was used for laser frequency stabilization using the PoundDrever-Hall technique. Here, an extended cavity diode laser was frequency tuned on the PMC acetylene P9 line exhibiting a free running fractional frequency instability (i.e. Allan variance) represented in fig. 5 (yellow curve) and compared when it is locked to the PMC P9 SA line (blue curve). The results show a factor 50 of improvement with an Allan variance of $2.10^{-10}$ for 100 s integration time proving the PMC is suitable for laser locking.
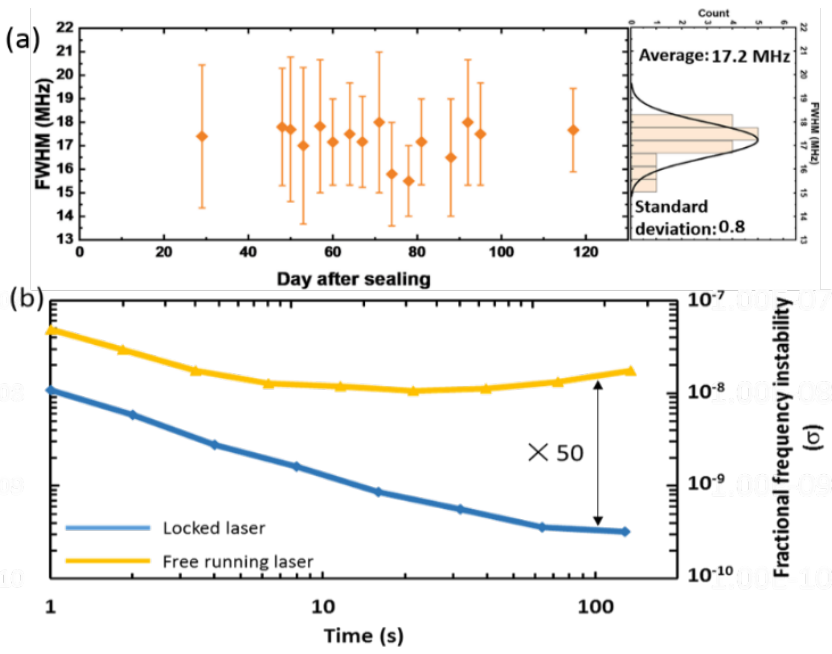

Fig. 5. (a) Evolution of SA linewidth during 95 days with a pump power of $23 \mathrm{~mW}$. Day zero corresponds to the day when the PMC is fully sealed. (b) Fractional frequency instability measurement for free running laser (yellow curve) and for locked laser with our PMC (blue curve).

In conclusion, we reported on a new end-cap technique to fabricate 7 meter long single mode PMC filled with $80 \mu$ bar of acetylene. The technique immunity from contamination and sealing power were demonstrated using sub-Doppler spectroscopy over a period of more than 3 months. The linewidth of the EIT and SA lines shows a FWHM of $18 \pm 2$ $\mathrm{MHz}$, in consistency with the gas pressure. We have used the PMC to frequency stabilize a laser, and achieved an Allan variance of $2.10^{-10}$ at 100 s integration time. The results indicate that this PMC assembly technique is ideal for compact and mobile frequency reference and quantum sensing applications.

Funding. Region Nouvelle Aquitaine.

Disclosures. The authors declare no conflicts of interest.

\section{References}

1. F. Benabid, F. Couny, J. C. Knight, T. A. Birks, and P. S. J. Russell, Nature 434, 488 (2005).

2. K. Knabe, S. Wu, J. Lim, K. A. Tillman, P. S. Light, F. Couny, N. Wheeler, R. Thapa, A. M. Jones, J. W. Nicholson, B. R. Washburn, F. Benabid, and K. L. Corwin, Opt. Express 17, 16017 (2009).

3. M. Triches, A. Brusch, and J. Hald, Appl. Phys. B Lasers Opt. 121, 251 (2015).

4. P. S. Light, F. Couny, and F. Benabid, Opt. Lett. 31, 2538 (2006).

5. F. Couny, P. S. Light, F. Benabid, and P. S. J. Russell, Opt. Commun. 263, 28 (2006).

6. E. A. Curtis, T. Bradley, G. P. Barwood, C. S. Edwards, N. V. Wheeler, R. Phelan, D. J. Richardson, M. N. Petrovich, and P. Gill, Opt. Express 26, 28621 (2018).

7. C. Wang, N. V. Wheeler, C. Fourcade-Dutin, M. Grogan, T. D. Bradley, B. R. Washburn, F. Benabid, and K. L. Corwin, Appl. Opt. 52, 5430 (2013).

8. N. V. Wheeler, M. D. W. Grogan, P. S. Light, F. Couny, T. A. Birks, and F. Benabid, Opt. Lett. 35, 1875 (2010).

9. N. V. Wheeler, P. S. Light, F. Couny, and F. Benabid, Opt. InfoBase Conf. Pap. 28, 870 (2009).

10. S. Hosseini-Zavareh, R. Luder, M. Thirugnanasambandam, H. W. Kushan Weerasinghe, B. R. Washburn, and K. L. Corwin, Appl. Opt. 58, 2809 (2019).

11. B. Debord, F. Amrani, L. Vincetti, F. Gérôme, and F. Benabid, Fibers 7, (2019).

12. I. E. Gordon, L. S. Rothman, et al. , J. Quant. Spectrosc. Radiat. Transf. 203, 3 (2017).

13. F. Amrani, J. H. Osório, F. Delahaye, F. Giovanardi, L. Vincetti, B. Debord, F. Gérôme, and F. Benabid, arXiv Prepr. arXiv2006.06375 (2020).

14. F. Benabid, P. S. Light, F. Couny, and P. S. J. Russell, Opt. Express 13, 5694 (2005).

15. J. Gea-Banacloche, Y.-Q. Li, S.-Z. Jin, and M. Xiao, Phys. Rev. a 51, (1995).

16. K. Shimoda, N. Bloembergen, V. P. Chebotayev, J. L. Hall, S. Haroche, P. Jacquinot, V. S. Letokhov, M. D. Levenson, and J. A. Magyar, Springer-V (1976).

17. B. Debord, M. Alharbi, T. Bradley, C. Fourcade-Dutin, Y. Y. Wang, L. Vincetti, F. Gérôme, and F. Benabid, Opt. Express 21, 28597 (2013). 
1. F. Benabid, F. Couny, J. C. Knight, T. A. Birks, and P. S. J. Russell, "Compact, stable and efficient all-fibre gas cells using hollow-core photonic crystal fibres, "Nature 434, 488491 (2005).

2. K. Knabe, S. Wu, J. Lim, K. A. Tillman, P. S. Light, F. Couny, N. Wheeler, R. Thapa, A. M. Jones, J. W. Nicholson, B. R. Washburn, F. Benabid, and K. L. Corwin, "10 kHz accuracy of an optical frequency reference based on $\mathrm{C}_{2} \mathrm{H} 2$-filled largecore kagome photonic crystal fibers, "Opt. Express 17, 16017 (2009).

3. M. Triches, A. Brusch, and J. Hald, "Portable optical frequency standard based on sealed gas-filled hollow-core fiber using a novel encapsulation technique, "Appl. Phys. B Lasers Opt. 121, 251-258 (2015).

4. P. S. Light, F. Couny, and F. Benabid, "Low optical insertionloss and vacuum-pressure all-fiber acetylene cell based on hollow-core photonic crystal fiber," Opt. Lett. 31, 2538 (2006).

5. F. Couny, P. S. Light, F. Benabid, and P. S. J. Russell, "Electromagnetically induced transparency and saturable absorption in all-fiber devices based on $12 \mathrm{C} 2 \mathrm{H} 2$-filled hollow-core photonic crystal fiber," Opt. Commun. 263, 2831 (2006).

6. E. A. Curtis, T. Bradley, G. P. Barwood, C. S. Edwards, N. V. Wheeler, R. Phelan, D. J. Richardson, M. N. Petrovich, and P. Gill, " Laser frequency stabilization and spectroscopy at $2051 \mathrm{~nm}$ using a compact CO 2 -filled Kagome hollow core fiber gas-cell system, "Opt. Express 26, 28621 (2018).

7. C. Wang, N. V. Wheeler, C. Fourcade-Dutin, M. Grogan, T. D. Bradley, B. R. Washburn, F. Benabid, and K. L. Corwin, "Acetylene frequency references in gas-filled hollow optical fiber and photonic microcells, "Appl. Opt. 52, 5430-5439 (2013).

8. N. V. Wheeler, M. D. W. Grogan, P. S. Light, F. Couny, T. A. Birks, and F. Benabid, "Large Core Acetylene-Filled Photonic Microcells Made by Tapering the Hollow-Core Fiber, "Opt. Lett. 35, 1875-1877 (2010).

9. N. V. Wheeler, P. S. Light, F. Couny, and F. Benabid, "Slow and superluminal light pulses via EIT in a 20-metre acetylene-filled photonic microcell," Opt. InfoBase Conf. Pap. 28, 870-875 (2009).

10. S. Hosseini-Zavareh, R. Luder, M. Thirugnanasambandam, H. W. Kushan Weerasinghe, B. R. Washburn, and K. L. Corwin, "Fabrication and characterization of short acetylene-filled photonic microcells, "Appl. Opt. 58, 2809 (2019).

11. B. Debord, F. Amrani, L. Vincetti, F. Gérôme, and F. Benabid, "Hollow-core fiber technology: The rising of "gas photonics,"" Fibers 7, (2019).

12. I. E. Gordon, L. S. Rothman, C. Hill, R. V. Kochanov, Y. Tan, P. F. Bernath, M. Birk, V. Boudon, A. Campargue, K. V. Chance, B. J. Drouin, J. M. Flaud, R. R. Gamache, J. T. Hodges, D. Jacquemart, V. I. Perevalov, A. Perrin, K. P. Shine, M. A. H. Smith, J. Tennyson, G. C. Toon, H. Tran, V. G. Tyuterev, A. Barbe, A. G. Császár, V. M. Devi, T. Furtenbacher, J. J. Harrison, J. M. Hartmann, A. Jolly, T. J. Johnson, T. Karman, I. Kleiner, A. A. Kyuberis, J. Loos, O. M. Lyulin, S. T. Massie, S. N. Mikhailenko, N. Moazzen-Ahmadi, H. S. P. Müller, O. V. Naumenko, A. V. Nikitin, O. L. Polyansky, M. Rey, M. Rotger, S. W. Sharpe, K. Sung, E. Starikova, S. A. Tashkun, J. Vander Auwera, G. Wagner, J. Wilzewski, P. Wcisło, S. Yu, and E. J.
Zak, "The HITRAN2016 molecular spectroscopic database," J. Quant. Spectrosc. Radiat. Transf. 203, 3-69 (2017).

13. F. Amrani, J. H. Osório, F. Delahaye, F. Giovanardi, L. Vincetti, B. Debord, F. Gérôme, and F. Benabid, "Low-loss single-mode hybrid-lattice hollow-core photonic crystal fiber," arXiv Prepr. arXiv2006.06375 (2020).

14. F. Benabid, P. S. Light, F. Couny, and P. S. J. Russell, "Electromagnetically-induced transparency grid in acetylene-filled hollow-core PCF," Opt. Express 13, 5694 (2005).

15. J. Gea-Banacloche, Y.-Q. Li, S.-Z. Jin, and M. Xiao, "EIT in ladder-type inhomogeneously broadened media: Theory and experiment," Phys. Rev. a 51, (1995).

16. K. Shimoda, N. Bloembergen, V. P. Chebotayev, J. L. Hall, S. Haroche, P. Jacquinot, V. S. Letokhov, M. D. Levenson, and J. A. Magyar, High-Resolution Laser Spectroscopy, Springer-V (1976).

17. B. Debord, M. Alharbi, T. Bradley, C. Fourcade-Dutin, Y. Y. Wang, L. Vincetti, F. Gérôme, and F. Benabid, "Hypocycloidshaped hollow-core photonic crystal fiber Part I: Arc curvature effect on confinement loss, "Opt. Express 21, 28597 (2013). 